\title{
Nova espécie de Heleobia (Rissooidea, Hydrobiidae) da planície costeira do sul do Brasil ${ }^{1}$
}

\author{
Maria Cristina Pons da Silva² \& Inga Ludmila Veitenheimer-Mendes ${ }^{3}$ \\ 1. Parte da tese de doutorado, da autora sênior, Programa de Pós-graduação em Biologia Animal (PPG-BAN), Universidade \\ Federal do Rio Grande do Sul (UFRGS). Contribuição n 411 do Departamento de Zoologia, UFRGS. \\ 2. Universidade Luterana do Brasil (ULBRA), Rua Miguel Tostes, 101, 92420-280, Canoas, RS, Brasil. (criss@ulbra.br) \\ 3. PPG-BAN, Departamento de Zoologia, UFRGS, Laboratório de Malacologia, Av. Bento Gonçalves, 9.500, prédio 43435, 91501 - \\ 970, Porto Alegre, RS, Brasil. (inga.mendes@ufrgs.br)
}

\begin{abstract}
New species of Heleobia (Rissooidea, Hydrobiidae) from south plain coast of Brazil. Heleobia robusta, new species from plain coast of Rio Grande do Sul, Brazil, is described. Conchology, conchometry, soft-part morphology including head-food, pallial structures, radula and some aspects of the female and male reproductive systems are provided.
\end{abstract}

KEYWORDS. Mollusca, Gastropoda, Prosobranchia, new species, Neotropical.

\section{INTRODUÇÃO}

Heleobia Stimpson, 1865 ( Hydrobiidae), de acordo com Hershler \& THOMPson (1992), compreende 101 espécies distribuídas na América do Sul, Europa, nas regiões mediterrâneas da África, e do mar Cáspio da Ásia. Dentre os sete gêneros de Hydrobiidae representados no continente sul-americano, Heleobia é o que apresenta maior diversidade e distribuição com 71 espécies.

Segundo CHOMENKo \& SCHÄFER (1984a, b), LANZER \& SCHäFER (1985) e LANZER (2001) é alta a ubiqüidade de representantes da família Hydrobiidae na bacia do rio Tramandaí, Rio Grande do Sul, Brasil. Martens (1868), Kleerekoper $(1944,1949,1955)$, Marcus \& Marcus (1963), Chomenko \& SchäFer (1984a, b), LANZer \& SCHÄFER (1985), SiLVA (1993) e LANZER (2001) registram cinco espécies para a planície costeira do Rio Grande do Sul a seguir citadas com suas respectivas localidadestipo: Heleobia australis (Orbigny, 1835), Montevideo, Uruguai; $H$. parchappei (Orbigny, 1835), Argentina; $H$. charruana (Orbigny, 1840), Uruguai; Potamolithus catharinae Pilsbry, 1911, Ibirama, Santa Catarina, Brasil; e Potamolithus ribeirensis Pilsbry, 1911, Iporanga, São Paulo, Brasil. É proposta uma nova espécie de Heleobia para a planície costeira do Rio Grande do Sul.

\section{MATERIAL E MÉTODOS}

Foram examinados topótipos de Heleobia charruana, incluídos na coleção do Museu de Ciências Naturais, Fundação Zoobotânica do Rio Grande do Sul (MCNZ): Uruguai, Departamento Canelones, Montevideo, Pocitos, Rio de La Plata, 23.I.1983, M.C.P. Silva \& G.M. Davis col., 961 exs (MCNZ 7802). O material-tipo da nova espécie encontra-se depositado no Departamento de Zoologia, Universidade Federal do Rio Grande do Sul (UFRG, curadora I. L. V. Mendes) e MCNZ, curadora V. L. L. Pitoni.

A seleção e a terminologia dos caracteres descritivos têm como base Hershler \& Ponder (1998). Para a conquiliometria seguiu-se Hershler \& LANDYe
(1988); para a contagem das voltas adotou-se HERSHLER \& PONDER (1998, fig. 17m); as medidas foram obtidas a partir de desenhos através de câmara lúcida acoplada a estereomicroscópio, com ocular dotada de retículo micrometrado. A análise estatística restringe-se ao cálculo das médias, desvios padrões e teste " $t$ " para verificação de dimorfismo sexual quanto às dimensões da concha. Concha, opérculo e rádula foram limpos com hipoclorito de sódio e fotomicrografados ao microscópio eletrônico de varredura (MEV) do Centro de Microscopia Eletrônica da UFRGS. O holótipo e o alótipo foram fotomicrografados com câmara digital, acoplada ao estereomicroscópio. As observações morfológicas das partes moles foram orientadas segundo Silva \& Veitenheimer-Mendes (2004). As informações morfométricas são fornecidas em milímetros ( $\mathrm{mm})$.

\section{Heleobia robusta sp. nov.} (Figs. 1-19)

Littoridina charruana; KLEEREKOPER, 1944:107; 1949:22; 1955:562; Marcus \& Marcus, 1963:46; 1965:72; Сhomenko \& SCHÄFER,1984a:162; 1984b:139 (non Orbigny,1840:384, est. 75, figs. 1-3)

Etimologia. Latim: robusta $=$ robusto, sólido. Relativo à parede espessada da concha.

Diagnose. Heleobia robusta difere das demais espécies do gênero por apresentar concha oval-cônica, espessada, ausência de depressão umbilical, lábio interno espessado, ângulo palatal agudo; bolsa copuladora dobrada, posicionada paralelamente ao ducto; glândula de albume junto à parede posterior da cavidade palial; espermioducto não dilatado; diâmetro do receptáculo seminal igual ao do ducto; glândula da próstata quase totalmente visceral.

Material-tipo. Holótipo $\subsetneq$ (UFRG 1317), alótipo 8 (UFRG 1318), BRASIL, Rio Grande do Sul: Pinhal, Lagoa Rondinha, 30.VI.2001, M.C.P. Silva \& J.A. Nunes col. Parátipos: Lagoa Itapeva, 16.II.2002, C. Cenzano col., 29 exs (UFRG 1436); Lagoa dos Quadros, 04.VII.1979, R.M. Lanzer col., 100 exs (MCNZ 
36401); Lagoa das Malvas, 18.IX.1991, R.M. Lanzer col., 27 exs (MCNZ 36404); Lagoa Pinguela, 09.IV.1980, R.M. Lanzer col., 4 exs (MCNZ 36406); Lagoa Pinguela, 27.VIII.1997, J.E.A. Caon col., 11 exs (UFRG 273); Lagoa Marcelino Ramos, VI.1979, L. Chomenko col., 13 exs (MCNZ 36407); Lagoa Palmital, 23.IV.1981, R.M. Lanzer col., 08 exs (MCNZ 36405); Lagoa Lessa, VIII.1979, L. Chomenko col., 20 exs (MCNZ 36402); 13.XI.1978, L. Chomenko col., 122 exs (MCNZ 36403); Lagoa Tramandaí, 09.IV.1988, R.M. Lanzer col., 35 exs (MCNZ 36408); Lagoa Gentil, 25.III.1984, R.M. Lanzer col., 10 exs (MCNZ 36409); Lagoa Manuel Nunes, 21.VIII.1981, R.M. Lanzer col. 12 exs (MCNZ 36413); Lagoa Fortaleza, II.1980, R.M. Lanzer col., 8 exs (MCNZ 36411); Lagoa Rondinha, 30.VI.2001, M.C.P. Silva \& J. A. Nunes col., 11700 exs (UFRG 1315); 13.I.1978, R.M. Lanzer col., 63 exs (MCNZ 34704); 18.II.1978, R.M. Lanzer col., 2 exs (MCNZ 36410); 05.V.2001, M.C.P. Silva \& J. A. Nunes col., 200 exs (UFRG 1322); Lagoa Cerquinha, 13.III.1981, R.M. Lanzer col., 29 exs (MCNZ 36412); Lagoa Rincão das Éguas, 31.VII.1979, R.M. Lanzer col., 12 exs (MCNZ 36627); Lagoa Cipó, 06.III.1981, R.M. Lanzer col., 2 exs (MCNZ 36630); Lagoa da Porteira, 31.VII.1979, R.M. Lanzer col., 2 exs (MCNZ 36628); Lagoa Capão Alto, 07.III.1981, R.M. Lanzer col., 100 exs (MCNZ 36631); Lagoa Charqueadas, 26.I.1981, R.M. Lanzer col., 30 exs (MCNZ 36629); canal para Quintão, 10.III.1986, M.C.P. Silva col., 18 exs (MCNZ 36624); Lagoa Barro Velho, 30.XII.1978, R.M. Lanzer col., 2 exs (MCNZ 36625); 08.III.1986, R.M. Lanzer col., 18 exs (MCNZ 36634); Lagoa Veiana, 20.II.1981, R.M. Lanzer col., 17 exs (MCNZ 36632); Lagoa Mirim, 13.II.1981, R.M. Lanzer \& A. Schäfer col., 32 exs (MCNZ 34711); Lagoa Nicola, 15.VIII.1980, R.M. Lanzer col., 5 exs (MCNZ 36626); 07.I.1986, M.C.P. Silva \& N. Schröder col., 1100 exs (MCNZ 9845); Lagoa Jacaré, 27.XI.1980, R.M. Lanzer col., 2 exs (MCNZ 34706); 13.II.1981, R.M. Lanzer col., 50 exs (MCNZ 36633).

Descrição. Concha (figss. 1-8) oval-cônica, contorno convexo, perióstraco castanho, freqüentemente com deposição de material enegrecido; sem perióstraco a cor é branca; em adultos, comprimento de 4,26 a 5,93; o número de voltas varia de 6 a 7 (tab. I); protoconcha lisa, achatada, com cerca de 1,5 voltas; teleoconcha lisa, linhas de crescimento fracamente marcadas, a partir da segunda volta podem apresentar linhas espirais, nem sempre presentes em todas as voltas (fig. 8); voltas convexas. Abertura oval, peristômio completo, lábio externo simples com parede espessa, prosocline; lábio interno espessado; umbílico ou depressão umbilical ausente. Há dimorfismo sexual quanto às dimensões da concha, com exceção da largura (tab. I).

Opérculo (figs. 9,10) com cerca de 1,8 de comprimento e 1,0 de largura; alongado-helicoidal, paucispiral, núcleo submarginal, borda interna convexa; plano, fino, flexível, de cor âmbar uniforme; superfícies, tanto dorsal como ventral lisas, marcadas apenas por indistintas linhas de crescimento; borda da cicatriz do músculo de inserção diferenciada, não espessada.

Cabeça-pé (figs. 11,12). Probóscide e nuca com pigmento negro, tentáculos e dorso do pé variam do cinzaescuro ao cinza-claro. A base dos tentáculos, o lábio, a faixa lateral entre a cabeça e o pé, e a sola do pé, desprovidos de pigmento. Probóscide, mais longa do que larga, apresenta a extremidade distal não afilada, lóbulos distais médios. Tentáculos cefálicos com uma banda longitudinal estreita totalmente despigmentada, tanto na face dorsal como ventral; contorno dos lados paralelos, extremidade distal arredondada, não dilatada; lóbulo da base pouco proeminente. Grânulos brancos dispersos ao longo dos tentáculos, agrupados na base, atrás dos
Tabela I. Dimensões da concha $(\mathrm{mm})$ de 10 machos, de 6 a 7 voltas, e 10 fêmeas, de 6 a 6,5 voltas, de Heleobia robusta (DSP $\leq 0,05$, diferença entre machos e fêmeas para um nível de $5 \%$ de significância; $\bar{X}$, média; DP, desvio padrão; entre parênteses, valor máximo e valor mínimo da amostra).

\begin{tabular}{lccc}
\hline Caracteres & $\begin{array}{c}\text { Fêmea } \\
\bar{X} \pm \mathrm{DP}\end{array}$ & $\begin{array}{c}\text { Macho } \\
\bar{X} \pm \mathrm{DP}\end{array}$ & DSP $\leq 0,05$ \\
\hline Comprimento & $4,63 \pm 0,32$ & $5,14 \pm 0,49$ & \multirow{2}{*}{$(4 \mathrm{sim}$} \\
& $(4,26-5,30)$ & $(4,68-5,93)$ & \\
Largura & $3,12 \pm 0,20$ & $3,25 \pm 0,16$ & \multirow{2}{*}{ não } \\
Comprimento da & $3,40 \pm 0,19$ & $3,68 \pm 0,18$ & \multirow{2}{*}{$\operatorname{sim}$} \\
volta do corpo & $(3,12-3,80)$ & $(3,38-3,95)$ & \\
Comprimento & $2,57 \pm 0,17$ & $2,85 \pm 0,26$ & \multirow{2}{*}{$\operatorname{sim}$} \\
da abertura & $(2,34-2,96)$ & $(2,65-3,55)$ & \\
Largura da & $1,88 \pm 0,11$ & $2,03 \pm 0,10$ & \multirow{2}{*}{$\operatorname{sim}$} \\
abertura & $(1,72-2,08)$ & $(1,92-2,18)$ & \\
\hline
\end{tabular}

olhos e, às vezes, também, na extremidade distal. Cílios predominam no tentáculo esquerdo, com bandas transversais. Região lateral, entre a cabeça e o pé, simples, sem sulco omnifórico ou qualquer outra estrutura lobular. Lobo nucal ausente nas fêmeas. Pé, alongado, com propódio completamente sopreposto à borda anterior côncava, cujas expansões laterais são fortemente desenvolvidas; borda posterior arredondada; dobra supra pediosa, ruga pediosa e tentáculo metapodial ausentes. Lóbulo do opérculo ultrapassa as bordas laterais do pé.

Cavidade palial. Borda do manto lisa e estreita, com pequeno espessamento pontiagudo na porção láteroventral direita que corresponde ao ângulo palatal. Grânulos brancos distribuídos ao longo da borda do manto formando uma faixa com cerca de 0,1 de largura. Ausência de tentáculos paliais. Teto da cavidade palial translúcido, sendo possível observar o pigmento negro da base dos filamentos branquiais. Ctenídio estende-se ao longo do teto da cavidade palial, desde cerca de 0,23 da borda do manto até junto ao pericárdio, na extremidade posterior; número de filamentos branquiais variou de 38 a 44 em 18 exemplares examinados; largura da base de cada filamento é maior do que o seu comprimento, estando a extremidade distal voltada para a direita. Osfrádio oval, sem pigmento, localizado na base da porção mediana do ctenídio; comprimento, paralelo ao eixo do ctenídio, inferior ao dobro da largura e inferior a $24 \%$ do comprimento do ctenídio. Ânus simples, a uma distância em torno de 0,30 da borda palial.

Sistema digestório. Comprimento da massa bucal oscila entre 0,84 a 0,97, a largura maior entre 0,44 a 0,49 e a altura 0,53 . De seis exemplares examinados $(2 \circ \mathrm{e} 4$ \& $)$ quatro apresentam glândulas salivares alongadas, em torno de 0,6 de comprimento e 0,12 de largura; duas fêmeas com glândulas salivares curtas, com 0,21 e 0,28 de comprimento e largura de 0,15 a 0,23 .

Rádula (figs. 14, 15). Comprimento da fita radular varia de 0,61 a 0,87 e largura de 0,15 a 0,19. Saco da rádula, não enrolado, posiciona-se abaixo e atrás da massa bucal e mede de 0,17 a 0,19 de comprimento. O número de fileiras de dentes na rádula varia de 51 a 59. Dente raquidiano trapezoidal, com 13 a 15 cúspides na borda cortante, cujo mediano destaca-se em tamanho em relação aos demais e tem extremidade distal afilada. Projeção central da base do dente é do tipo "V" estreito, sendo mais curta do que 

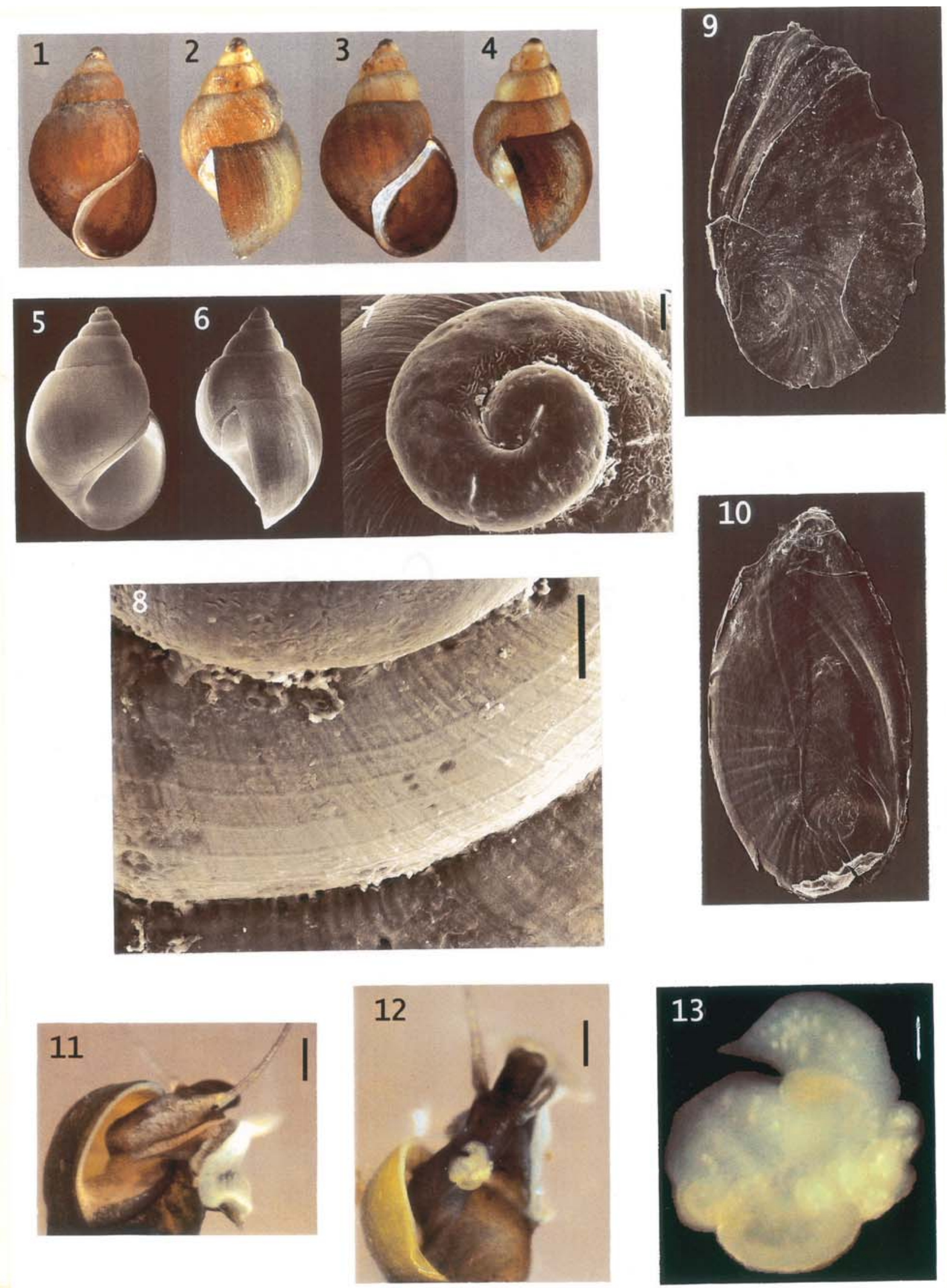

Figs. 1-13. Heleobia robusta sp. nov.: 1, 2, holótipo $\subsetneq$ (comprimento 5,24); 3, 4, alótipo $\zeta$ (comprimento 4,6); 5-8, parátipos (UFRG 1315): 5, vista frontal (comprimento 4,87); 6, vista lateral (comprimento 5,07); 7, protoconcha; 8, linhas espirais. 9-13, (UFRG 1315): 9, 10, opérculo: 9, face externa (comprimento 1,81); 10, face interna (comprimento 1,8); 11, cabeça e nuca de fêmea; 12, cabeça e nuca de macho; 13, pênis. Escalas: $40 \mu \mathrm{m}$, figs. 7, 8;0,7 mm, figs. 11, 12;0,1 mm, fig. 13 . 

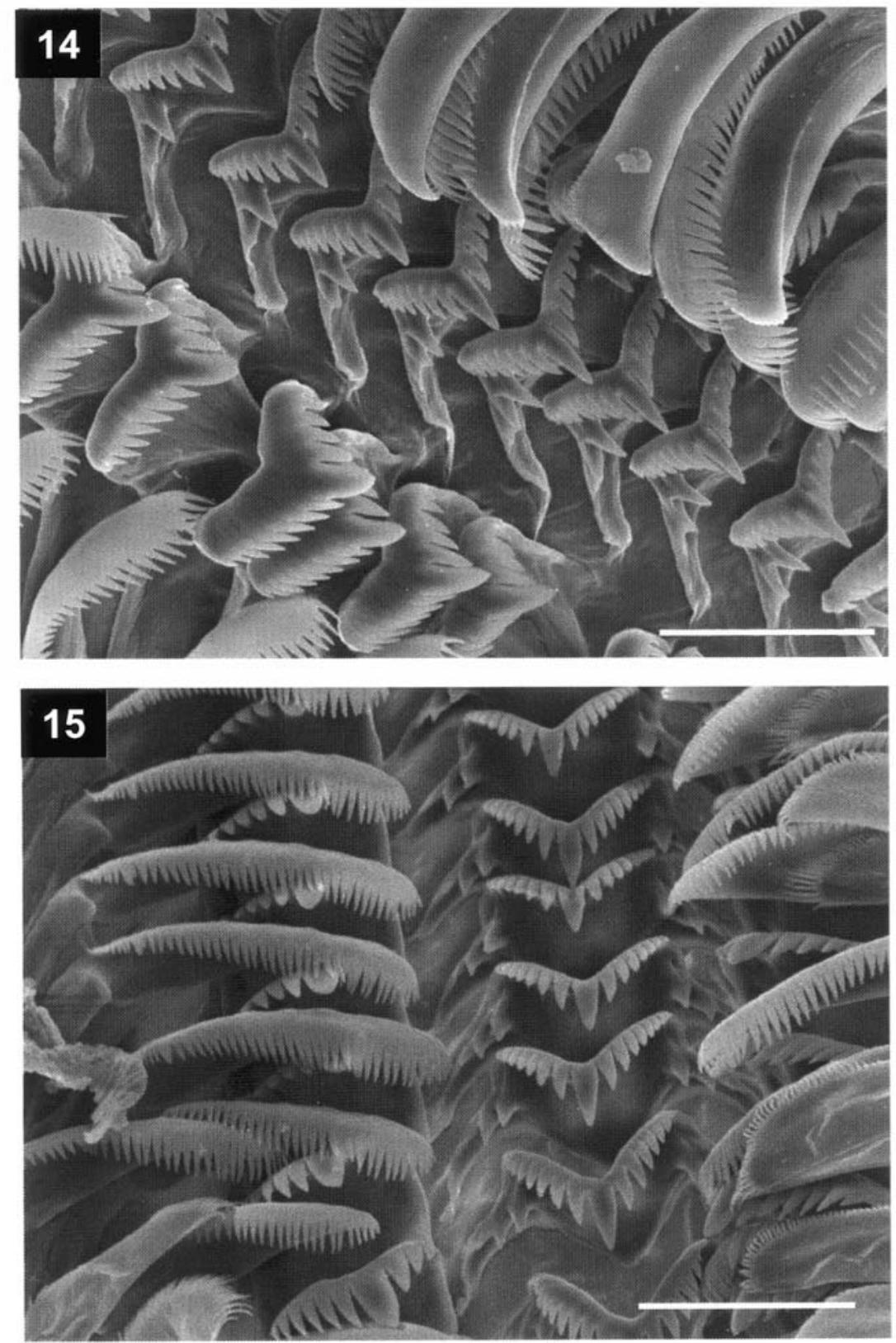

Figs. 14, 15. Heleobia robusta sp. nov. (UFRG 1315), rádula. Escalas: $10 \mu \mathrm{m}$.

as margens laterais. Ladeando a projeção central da base do dente raquidiano, há profundos arcos que ocupam cerca de $50 \%$ da altura total do dente. Cúspides basais variam de um a dois pares, situadas na porção superior do ângulo lateral e, quando há dois pares, estas são aproximadamente do mesmo tamanho. Dente lateral com curvatura acentuada na sua margem externa; fórmula 8$1-5$, tendo o mediano a extremidade afilada; forma da face quadrada e com projeção basal. Ausência de dentículo acessório. Dente marginal interno com borda cortante ocupando mais do que $25 \%$ da extensão do comprimento do dente; uma saliência pontiaguda entre a face do dente e seu pedúnculo. Cúspides na margem interna do dente, maiores que as da externa. $\mathrm{O}$ aspecto geral das demais estruturas do sistema digestório apresenta-se como já descrito em outras espécies do gênero.
Sistema reprodutor feminino (figs. 16, 17). O ovário se posiciona a cerca de duas voltas do ápice, ocupando de 1,5 a 2 voltas, seu limite proximal é o mesmo da glândula digestiva. Ovário ramificado, composto por conjuntos de lóbulos alongados $(570 \mu \mathrm{m}$ de altura e $46 \mu \mathrm{m}$ de largura), formando cachos. Ducto gonopericardial não observado. Oviduto não pigmentado, com no mínimo duas voltas antes de ligar-se à extremidade posterior do espermioducto, seguindo após em direção à glândula de albume. Voltas do oviduto no lado direito da glândula de albume. Receptáculo seminal único, alongado, aparentemente aderido à bolsa copuladora . Morfologicamente não é possível determinar o limite entre o receptáculo seminal e seu ducto. Espermioducto, continuação do ducto do receptáculo seminal, com largura um pouco inferior a do ducto da bolsa copuladora 

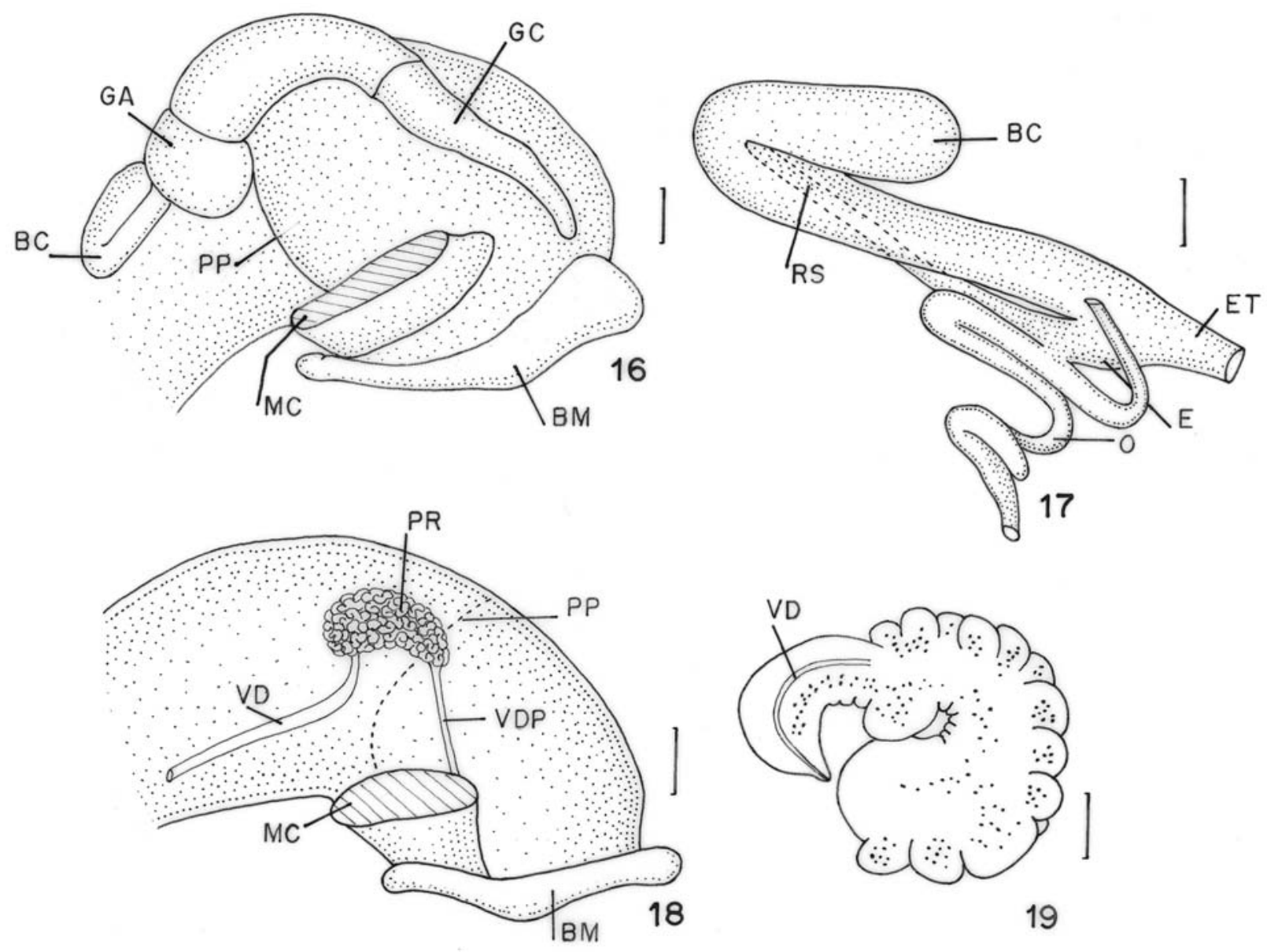

19

Figs. 16-19. Heleobia robusta sp. nov. (UFRG 1315): 16, 17, sistema reprodutor feminino: 16, posição da bolsa copuladora e do oviduto palial em relação à extremidade posterior da cavidade do palial; 17, complexo da bolsa copuladora, a partir da remoção da porção posterior do oviduto glandular; 18,19 , sistema reprodutor masculino:18, posição da glândula da próstata em relação à extremidade posterior da cavidade palial; 19, pênis. (BC, bolsa copuladora; BM, borda do manto; E, espermioducto; ET, espermiotubo; GA, glândula de albume; GC, glândula da casca; MC, músculo columelar; O, oviduto; PP, parede palial; PR, próstata; RS, receptáculo seminal; VD, vaso deferente; VDP, vaso deferente palial). Escalas: $0,17 \mathrm{~mm}$, fig. $16 ; 0,1 \mathrm{~mm}$, fig. $17 ; 0,4 \mathrm{~mm}$, fig. $18 ; 0,2 \mathrm{~mm}$, fig. 19.

e comprimento bem inferior, cerca de $1 / 4$ deste, sendo curto, porém não dilatado. Bolsa copuladora oval, dobrada sobre seu ducto, posicionada póstero-ventralmente à glândula de albume, levemente coberta por esta. $\mathrm{O}$ ducto da bolsa une-se ao espermioducto para formar um curto espermiotubo o qual se abre no fundo da cavidade palial. Glândula de albume, mais larga do que longa, logo atrás da parede posterior da cavidade palial. Glândula da casca, mais de $90 \%$ palial, cerca de cinco vezes mais longa do que a de albume, com duas áreas de tonalidades diferentes, porém equivalentes em comprimento.

Sistema reprodutor masculino (figs. 11-13, 18, 19). $\mathrm{O}$ vaso deferente parte da porção anterior do testículo e percorre retilineamente a base deste em direção posterior e, antes de atingir o ápice da massa visceral, dobra-se para frente e enovela-se originando a vesícula seminal que se estende até a área onde o esôfago liga-se ao estômago. Neste ponto, continua retilineamente cruzando a área ventral do estômago até receber a próstata. Glândula da próstata reniforme, posicionada atrás da parede posterior da cavidade palial, exceto a extremidade anterior de onde parte o vaso deferente palial, que segue retilineamente pela parede ventral do palio até penetrar na base do músculo columelar. Pênis, cuja base situa-se junto à linha média da região da nuca a menos de 0,5 da base do tentáculo direito, apresenta em seu lado convexo 9 a 13 glândulas apócrinas e, freqüentemente, um espessamento glandular de base larga; no lado côncavo há um lobo.

Distribuição. Brasil, Rio Grande do Sul, ao longo da planície costeira.

Hábitat. Em fundo arenoso, em vegetação aquática das lagoas costeiras.

Discussão. A concha assemelha-se à de $H$. charruana, por apresentar volta do corpo globosa e espira baixa, o que deve ter orientado à identificação equivocada. Os caracteres da concha, pênis e rádula fornecidos por MARCUS \& MARCUS (1965), para oito exemplares procedentes do rio Tramandaí, RS e identificados como Littoridina charruana, correspondem 
àqueles de $H$. robusta. Cabe ressaltar que o menor número de cúspides nos dentes da rádula, registrado por MARCUS \& MARCUs (1965), decorre de observações ao microscópio óptico.

As descrições de $H$. charruana encontradas em Orbigny (1840) e Silva \& Davis (1983), além do exame de exemplares procedentes da localidade-tipo (Montevideo, Uruguai), mostram que distingue-se de $H$. robusta por apresentar: concha com voltas mais aplanadas, lábio externo menos espessado, depressão umbilical; espermioducto dilatado, diâmetro do receptáculo seminal maior que aquele de seu ducto, bolsa copuladora não dobrada sobre seu ducto, pênis com glande pigmentada de negro e um grande lobo no lado côncavo, pigmentado de marrom na base e na margem posterior.

Heleobia robusta aproxima-se de $H$. davisi descrita por Silva \& Thomé (1985), entretanto difere por apresentar: concha com lábio externo fino; rádula com um número maior de fileiras de dentes e dentes raquidiano e lateral com menor número de cúspides na borda cortante; espermioducto dilatado e glândula da próstata palial.

Agradecimentos. Ao Dr. George M. Davis, Academy of Natural Sciences of Philadelphia, pela participação na coleta e estudo da anatomia de topótipos de $H$. charruana. À curadora da coleção MCNZ, pelo empréstimo dos lotes. Ao M. Sc. Fábio Wiggers, Laboratório de Malacologia (UFRGS), pelas fotomicrografias digitalizadas. À desenhista Rejane Rosa pela arte final dos desenhos.

\section{REFERÊNCIAS BIBLIOGRÁFICAS}

Chomenko, L. \& SchäFer, A. 1984a. Untersuchungen zur salzwassertoleranz bei arten des genus Littoridina (Hydrobiidae) der Küstenseen von Rio Grande do Sul. Biogeographica, Saabrücken, 19:161-173.

1984b. Interpretação biogeográfica da distribuição do gênero Littoridina (Hydrobiidae) nas lagoas costeiras do Rio Grande do Sul, Brasil. Amazoniana, Manaus, 9(1):127-146.

Hershler, R. \& Landye, J. J. 1988. Arizona Hydrobiidae (Prosobranchia: Rissoacea). Smithsonian Contributions to Zoology, Washington, 459:1-63.
Hershler, R. \& Ponder, W. F. 1998. A review of morphological characters of Hydrobioid snails. Smithsonian Contributions to Zoology, Washington, 600:1-55.

Hershler, R. \& Thompson, F. G. 1992. A review of the aquatic gastropod subfamily Cochliopinae (Prosobranchia: Hydrobiidae). Malacological Review, Ann Arbor, 5(supl.):1140 .

KLEEREKOPER, H. 1944. Introdução ao estudo da limnologia I. Rio de Janeiro, Ministério da Agricultura. 329 p.

1949. O peixe-rei. Serviço de Informática Agrícola. 2. ed., Rio de Janeiro, Ministério da Agricultura. 102p.

1955. Limnological observations in northeastern Rio Grande do Sul, Brazil I. Archiv fur Hydrobiologie, Stuttgart, 50(3/4):553-567.

LANZER, R. 2001. Distribuição, fatores históricos e dispersão de moluscos límnicos em lagoas do sul do Brasil. Biociências, Porto Alegre, 9(2):63-84.

LANZER, R. \& SchÄFER, A. 1985. Padrões de distribuição de moluscos dulciaquícolas nas lagoas costeiras do sul do Brasil. Revista Brasileira de Biologia, Rio de Janeiro, 45(4):535-545.

Marcus, E. \& Marcus, E. 1963. On Brazilian supralittoral and brackish water snails. Boletim do Instituto Oceanográfico, São Paulo, 25:19-82.

1965. On Brazilian supratidal and estuarine snails. Boletim da Faculdade de Filosofia Ciências e Letras, São Paulo, (25):19-82.

MARTENS, E. von. 1868. Ueber Südbrasilianische land und süsswasser - Mollusken. Malakozoologische Blätter, Cassel, 15:169217.

Orbigny, A. D'. 1840. Famille littorinidées, Littorinidae, d'Orb. In: Voyage dans l'Ámérique méridionale; mollusques. Paris, P. Bertrand. v. 5, pt. 3, p. 384, 385.

Silva, M. C. P. DA. 1993. Dados morfológicos de Heleobia parchappei (Orbigny, 1835) (Prosobranchia, Hydrobiidae, Littoridininae). Iheringia, Sér. Zool., Porto Alegre (75):81-87.

Silva, M. C. P. DA \& DAvis, G. M. 1983. D'Orbigny's type specimens of Paludestrina (Gastropoda: Prosobranchia) from southern South America. Proceedings of the Academy of Natural Sciences of Philadelphia, Philadelphia, 135:128146

Silva, M. C. P. dA \& Thomé, J. W. 1985. Uma nova Heleobia (Prosobranchia: Hydrobiidae) do "rio" Guaíba, Rio Grande do Sul. Revista Brasileira de Biologia, Rio de Janeiro, 45(4):515-534.

Silva, M. C. P. DA \& Veitenheimer-Mendes, I. L. 2004. Redescrição de Potamolithus catharinae Pilsbry, 1911 com base em topótipos (Gastropoda; Hydrobiidae). Iheringia, Sér. Zool., Porto Alegre, 94(1):83-88. 